\title{
Impacto y visibilidad de la Revista Chilena de Cirugía tras su indización en las bases de datos SciELO e ISI. Análisis bibliométrico*
}

\author{
Drs. RICARDO CARTES-VELÁSQUEZ V. ${ }^{1,2,3}$, JAVIER MORAGA C. ${ }^{1}$, \\ PEDRO ARAVENA T. ${ }^{1,4}$, CARLOS MANTEROLA D. ${ }^{1,5}$ \\ 1 Programas de Doctorado y Magíster en Ciencias Médicas, Universidad de la Frontera, Temuco. \\ Facultad de Odontología, Universidad de Concepción, Concepción. \\ Centro de I+D en Odontología Social, CIDOS.org. \\ 4 Facultad de Medicina, Universidad Austral de Chile, Valdivia. \\ 5 Departamento de Cirugía y Traumatología, Universidad de la Frontera, Temuco. \\ Chile.
}

\begin{abstract}
Impact and visibility of Revista Chilena de Cirugía after its indexing on SciELO and ISI databases. Bibliometric analysis
\end{abstract}

Aim: To characterize the international visibility and impact of Revista Chilena de Cirugía (RCC) after its indexing on SciELO (2006) and ISI (2009) databases. Methods: Search on WOK platform of ThomsonReuters for ISI citations to RCC articles in the pre-indexing (1992-2005), SciELO indexing (2006-2008) and ISI indexing (2009-2011) periods. Analyzed variables were: total number and anual average of cites to RCC, RCC articles cited and ISI citer articles to RCC; authors, journals and institutions who cite RCC and its international or national origin, and triennial cites. Results: Anual average of ISI cites for pre-indexing, SciELO indexing and ISI indexing periods were $16.9 ; 58.3$ y 164.7 , respectively; meanwhile the international origin of articles that cite RCC in the authors-institutions/publication level for each period were $8.6 \% / 32.4 \% ; 8.5 \% / 38.3 \%$; and $25.8 \% / 33.6 \%$, respectively; triennial cites for each period were 3,12 and 34 . Publications with more cites to RCC were Rev Med Chile, RCC, World J Surg and Cir Esp, meanwhile in authors-institutions level we saw a concentration on Universidad de Chile, Pontificia Universidad Católica and Universidad de La Frontera. Conclusion: SciELO and ISI indexing has brougth an increase of its impact (cites) and international visibility, although it persists a strong endogamic citation pattern that should be fixed in order to achieve a good international level

Key words: "Bibliometrics"[Mesh], Bibliometric Analysis, "Surgical Procedures, Operative"[Mesh], "surgery" [Subheading], Chile.

*Recibido el 25 de junio de 2012 y aceptado para publicación el 2 de agosto de 2012.

Los autores no refieren conflictos de interés.

Correspondencia: Dr. Carlos Manterola D.

Manuel Montt 112, oficina 408, Temuco, Chile. Fax: 56-45-325761

cmantero@ufro.c1 


\section{Resumen}

Objetivo: Caracterizar la visibilidad internacional e impacto de la Revista Chilena de Cirugía (RCC) tras su indización en las bases SciELO (2006) e ISI (2009). Método: Búsqueda en la plataforma WOK de Thomson-Reuters de las citaciones ISI de artículos de la RCC en el período pre-indización (1992-2005), indización SciELO (2006-2008), e indización ISI (2009-2011). Las variables analizadas fueron: número total y promedio anual de citas a la RCC, de artículos de la RCC citados y de artículos que citan a la RCC; autores, revistas e instituciones que citaron, así como su carácter (nacional e internacional), y las citas trienales. Resultados: El promedio anual de citas ISI en el período pre-indización, SciELO e ISI fue de 16,9; 58,3 y 164,7 , respectivamente; mientras que el grado de internacionalización de los artículos que originaron las citas a nivel de autores-instituciones/revistas para cada período fue de 8,6\% / 32,4\%; 8,5\% / 38,3\%; y $25,8 \% / 33,6 \%$, respectivamente; las citas trienales de cada período fueron 3, 12 y 34 . Las revistas con más citas a RCC fueron Rev Med Chile, RCC, World J Surg y Cir Esp, mientras que a nivel de autores-instituciones vemos una concentración en la Universidad de Chile, Pontificia Universidad Católica y Universidad de La Frontera. Conclusión: La indización de RCC en las bases SciELO e ISI ha traído un incremento en su impacto (citas) y visibilidad internacional, aunque persiste una fuerte citación endogámica que debe ser resuelta a fin de alcanzar un buen nivel internacional.

Palabras clave: Bibliometría, cirugía, Chile.

\section{Introducción}

Desde la aparición de la primera revista científica (Journal des Scavans en 1665, la primera auténticamente médica fue Nouvelles Découvertes en 1679) el objetivo principal de ellas ha sido la divulgación y dialéctica, promoviendo de este modo el desarrollo de la ciencia, ya sea para impactar positivamente a la sociedad o como muestra del valor de la ciencia por la ciencia ${ }^{1}$.

En el cumplimiento de estos objetivos comenzó a florecer hace más de 50 años un área dedicada al estudio de los "rendimientos" asociados al cumplimiento de dichos objetivos; ésta, conocida como bibliometría, ha permitido describir y explicar en términos cuantitativos y cualitativos cómo se produce la ciencia, específicamente en la etapa de la divulgación ${ }^{2}$.

Son los resultados de esta nueva ciencia, que estudia la producción científica, los que han creado una serie de indicadores, bases de datos y rankings que con el objetivo de determinar calidad/impacto/ utilidad, han venido en último término a generar una carrera por la excelencia científica, resumida en el célebre publish or perish, famoso aforismo de la academia estadounidense ${ }^{3}$ que cruza investigadores, instituciones y precisamente a las revistas científicas. En el caso de estas últimas, la calidad se ha visto asociada a la indización a colecciones o bases de datos como SciELO, MEDLINE, SCOPUS e ISI (actual Thomson-Reuters), posteriormente la medición de su factor de impacto (calidad) a través del número de citas que obtienen sus artículos para un período determinado y el grado de visibilidad internacional (reconocimiento) que se asocia con dicho impacto ${ }^{4,5}$.
La Revista Chilena de Cirugía (RCC) ha mostrado una maduración consistente a través de su indización en la colección SciELO el año $2006^{6}$ y en la base de datos ISI el $2009^{7}$, cumpliendo con su rol de divulgación, dialéctica y desarrollo científico en el ámbito quirúrgico; sin embargo, aún no existen reportes bibliométricos que den cuenta de sus últimos progresos. El objetivo del presente estudio es determinar los cambios sufridos por la RCC en términos de impacto y visibilidad (internacional) tras su indización en SciELO e ISI.

\section{Material y Método}

\section{Diseño}

Estudio bibliométrico.

\section{Población a estudio}

Publicaciones indizadas en las últimas dos décadas (enero de 1992 a diciembre de 2011) en la base de datos Science Citation Index Expanded (SCI-E) de Thomson-Reuters (ISI) que entre sus citas consideraran a artículos de la RCC.

\section{Estrategia de búsqueda}

Se realizó una búsqueda avanzada de citas (Cited Reference Search) y de artículos (Advanced Search) a partir de la plataforma Web of Knowledge (WOK, http://apps.webofknowledge.com, el 26 de mayo de 2012, utilizando el título corto de RCC, "Rev Chil Cir"; la que arrojó la nómina que artículos ISI que citaron a la RCC en el período en estudio.

\section{Variables estudiadas}

Se determinaron las variables: número total y promedio anual de artículos de la RCC citados en 
ISI; de citas ISI a artículos de la RCC y de artículos ISI que citaron a la RCC; autores, instituciones y revistas que originaron las citas, así como el carácter nacional o internacional de estas últimas tres variables. Se determinó además el número de citas ISI del último año de cada período estudiado para artículos de la RCC publicados en los 2 años previos a dicho año, tal y como se calcula el Factor de Impacto Thomson-Reuters ${ }^{5}$, pero sin dividirlo por el total de artículos en el bienio mencionado, determinando así una variable que denominamos citas trienales.

\section{Plan de análisis}

Se realizó un "Citation Report" y sucesivos "Analize Results" en la plataforma WOK. Para facilitar una visión comparativa se realizó un análisis de los períodos: pre-indización (1992-2005), indización SciELO (2006-2008), e indización ISI (2009-2011). Se aplicó estadística descriptiva. Los datos fueron tabulados y tratados en una planilla MS Excel 2003; a partir de la cual se aplicó estadística descriptiva.

\section{Resultados}

En la Tabla 1 se puede apreciar la caracterización cuantitativa de las citaciones a la RCC para los períodos en estudio. En las Tablas 2, 3 y 4 podemos apreciar a los autores, revistas e instituciones que más citas han generado a artículos de la RCC.
Al considerar un análisis de la visibilidad internacional vemos que de los 105 artículos que citaron a la RCC en el período de pre-indización, 96 de ellos tenían participación de autores e instituciones chilenas; vale decir, la citación internacional en este aspecto fue de un $8,6 \%$, en tanto que 71 artículos provenían de la Revista Médica de Chile y el resto de publicaciones extranjeras, o sea, un $32,4 \%$ de internacionalización en este sentido.

Al observar el segundo período en estudio (indización en SciELO), se verifica que la internacionalización por autor/institución fue de $8,5 \%$ y a nivel de publicación de un 38,3\%; es decir, de los 47 artículos encontrados, 43 tenían participación de autores e instituciones chilenas y a nivel de publicaciones 28 fueron en la Revista Médica de Chile y 1 en la Revista Chilena de Infectología.

Para el período de indización ISI, se constató un $25,8 \%$ de internacionalización. Es decir que sobre un total de 217 artículos, 161 tuvieron participación de autores e instituciones chilenas, y a nivel de publicaciones un $33,6 \%$, con un total de 144 citas provenientes de revistas nacionales, con 107 originados desde la propia RCC. Si excluimos los artículos de la RCC del cálculo, los niveles de internacionalización alcanzan al $49,1 \%$ a nivel de autores e instituciones y al 57,3\% a nivel de publicaciones.

Finalmente, las citas trienales para el período de pre-indización fueron de 3, para el de indización SciELO de 12 y para el de indización ISI de 34.

Tabla 1. Citas de artículos ISI a la RCC, 1992-2011

\begin{tabular}{|lccc|}
\hline Período & $\begin{array}{c}\text { Artículos RCC citados } \\
\text { Total / anual }\end{array}$ & $\begin{array}{c}\text { Artículos que citan a RCC } \\
\text { Total / anual }\end{array}$ & $\begin{array}{c}\text { Total de citas a artículos RCC } \\
\text { Total / anual }\end{array}$ \\
Pre-indización & $130 / 9,3$ & $105 / 7,5$ & $236 / 16,9$ \\
Indización SciELO & $71 / 23,7$ & $47 / 15,7$ & $175 / 58,3$ \\
Indización ISI & $286 / 95,3$ & $217 / 72,3$ & $494 / 164,7$ \\
Global & $430 / 21,5$ & $369 / 18,4$ & $680 / 34$ \\
\hline
\end{tabular}

Tabla 2. Autores que más citan a la RCC, 1992-2011

\begin{tabular}{|lclclc|}
\hline \multicolumn{2}{c}{ Pre-indización } & \multicolumn{2}{c}{ Indización ScieLO } \\
Autor & (n artículos) & Autor & artículos) & Autor & $\begin{array}{c}\text { Indización ISI } \\
\text { (n artículos) }\end{array}$ \\
Csendes A & $(22)$ & Csendes & $(7)$ & Manterola C & $(25)$ \\
Manterola C & $(14)$ & Beltrán MA & $(6)$ & Sanhueza A & $(9)$ \\
Burdiles P & $(13)$ & Burgos AM & $(5)$ & Vial M & $(9)$ \\
Muñoz S & $(13)$ & Cruces KS & $(5)$ & Bannura G & $(8)$ \\
Braghetto I & $(12)$ & Burdiles P & $(4)$ & Contreras G & $(8)$ \\
\hline
\end{tabular}


Tabla 3. Revistas que más citan a la RCC, 1992-2011

\begin{tabular}{|lclclc|}
\hline \multicolumn{2}{c}{ Pre-indización } & \multicolumn{2}{c}{ Indización ScieLO } & \multicolumn{2}{c|}{ Indización ISI } \\
Revista & (n artículos) & Revista & (n artículos) & Revista & (n artículos) \\
\hline Rev Med Chile & $(71)$ & Rev Med Chile & $(28)$ & Rev Chil Cir & $(107)$ \\
World J Surg & $(6)$ & World J Surg & $(3)$ & Rev Med Chile & $(25)$ \\
\hline Obes Surg & $(3)$ & Obes Surg & $(2)$ & Cir Esp & $(8)$ \\
J Clin Epidemiol & $(2)$ & Prevent Med & $(1)$ & Int J Morphol & $(7)$ \\
\hline J Gastroint Surg & $(2)$ & Rev Chil Infectol & $(1)$ & Cir Cir & $(5)$ \\
\hline
\end{tabular}

Tabla 4. Instituciones que más citan a la RCC, 1992-2011

\begin{tabular}{|lclclc|}
\hline \multicolumn{2}{c}{ Pre-indización } & \multicolumn{2}{c}{ Indización ScieLO } & \multicolumn{2}{c|}{ Indización ISI } \\
Institución & (n artículos) & Institución & (n artículos) & Institución & (n artículos) \\
\hline U Chile & $(37)$ & PUC Chile & $(16)$ & U Chile & $(49)$ \\
PUC Chile & $(21)$ & U Chile & $(13)$ & UFRO & $(38)$ \\
UFRO & $(20)$ & H Ovalle & $(5)$ & PUC Chile & $(29)$ \\
H Temuco & $(9)$ & H JJA & $(3)$ & U Austral Chile & $(7)$ \\
U y H & $(3)$ & U y H & $(3)$ & U Católica Norte & $(7)$ \\
\hline
\end{tabular}

U: Universidad. PUC: Pontificia Universidad Católica. UFRO: Universidad de La Frontera. H: Hospital. JJA: José Joaquín Aguirre.

\section{Discusión}

Los resultados objetivados muestran una tendencia clara de incremento en el número de citas (impacto) que recibe la RCC para el período global estudiado, especialmente al comparar los promedios de los períodos analizados, así como de la visibilidad internacional que implican dichas citas.

No obstante ello, es relevante mencionar que determinar las causas de este fenómeno está más allá de los objetivos de este estudio. Sin embargo, basándonos en la evidencia bibliométrica, podríamos plantear tres posibles causas, a analizar en futuros estudios, estas son: un incremento en la calidad metodológica (CM) de los artículos publicados en la RCC; puesto que, en revistas del ámbito quirúrgico se ha determinado una relación directa entre el factor de impacto y la $\mathrm{CM}^{8}$; un aumento de la valoración de la investigación científica y el interés por publicar de los cirujanos chilenos, relacionado al incremento de artículos ISI durante la década 2001-2010 con autoría de la comunidad quirúrgica nacional9; y la influencia del modelo de difusión electrónica, con la posibilidad de descarga gratuita del texto completo de artículos desde la colección SciELO; puesto que hay evidencia que respalda la tesitura que artículos disponibles a texto completo en Internet triplican la obtención de citas que aquellos en papel ${ }^{10}$, aunque no necesariamente a costa de una mayor calidad metodológica, sino simplemente por mayor accesibilidad $^{11,12}$.

Sin embargo, un alto porcentaje de las citas durante el período de indización ISI provienen de revistas ISI nacionales, como Revista Médica de Chile e International Journal of Morphology, y otras tantas de la propia RCC (autocitas); lo que representa un fenómeno endogámico que podría ser meta de la política editorial reducir en el próximo quinquenio generando estrategias que aumenten la visibilidad de RCC.

De modo similar, se ha de precisar que una proporción muy significativa de las citas a RCC desde revistas internacionales, son realizadas por autores chilenos, lo que puede asociarse por una parte a un reconocimiento colaborativo de los autores nacionales, relacionado a una preservación de la difusión científica en la propia lengua ${ }^{12}$; y por otra, a la pobre consideración y valoración a nivel internacional de los artículos publicados por la RCC por parte de grupos de investigación internacionales.

A pesar de lo anterior, debemos recalcar que "impacto" o "visibilidad" no es exactamente sinónimo de "calidad", ya que la medición más fidedigna de la calidad de una revista está dada por el análisis crítico de cada uno de sus artículos ${ }^{8,12}$ y no por la cantidad de artículos publicados en cada número o 
el diseño de estudio que incentiva a citar un mayor número de artículos, como es el caso de las revisiones bibliográficas, que de por sí obtiene más citas que los estudios originales ${ }^{13}$. Un claro ejemplo de aquello son los 100 artículos más citados por las revistas de cirugía, reportados entre los años 1945 y 1995, de los cuales 80 fueron reportes de experiencias clínicas, 6 artículos de revisión y sólo 14 de ciencias básicas ${ }^{14}$.

En resumen, observamos que la indización de la RCC en SciELO e ISI ha traído consigo un incremento en su impacto (citas) y visibilidad internacional, aunque persiste una fuerte citación endogámica que debe ser resuelta a fin de alcanzar un buen nivel internacional.

\section{Referencias}

1. López JA. Primera revista médica de América. ACIMED 2000;8:133-9.

2. Garfield E. Citation Indexes for Science: A New Dimension in Documentation through Association of Ideas. Science 1955:132(3159):105-11.

3. Lolas F, Quezada A, Rodríguez E. Investigación en Salud. Dimensión ética. Santiago: CIEB, Universidad de Chile, 2006;266.

4. Spreckelsen C, Deserno TM, Spitzer K. Visibility of medical informatics regarding bibliometric indices and databases. BMC Med Inform Decis Mak 2011;11:24.
5. Chattopadhyay A. How useful is journal impact factor? Indian J Dent Res. 2009;20:246-8.

6. Manterola C. Revista Chilena de Cirugía indexada en SciELO. Lo relevante y lo que viene por delante. Rev Chil Cir. 2007;59:1-2.

7. Yarmuch J. Revista Chilena de Cirugía en ISI. Cuál es nuestra tarea. Rev Chil Cir. 2010;62:107.

8. Manterola C, Pineda V, Vial M, Losada H. What Is the Methodologic Quality of Human Therapy Studies in ISI Surgical Publications? Ann Surg 2006;244:827-32.

9. Moraga J, Cartes-Velásquez R, Urrutia S, Manterola C. Publicaciones de autores chilenos en revistas quirúrgicas durante los últimos diez años. Rev Chil Cir. 2012;64:447-51.

10. Lawrence S. Free online availability substantially increases a paper's impact. Nature 2001;411:521.

11. MuraliI NS, Murali HR, Auethavekiat P, Erwin PJ, Mandrekar JN, Manek NJ, et al. Impact of FUTON and NAA bias on visibility of research. Mayo Clin Proc. 2004;79:1001-6.

12. Zárate V, Cerda J. Fortalezas y debilidades del factor de impacto de revistas científicas. Rev Med Chile 2007; 135:1474-8.

13. Seglen P. Why the impact factor of journals should not be used for evaluating research. BMJ 1997;314:498502.

14. Paladugu R, Schein M, Gardezi S, Wise L. One Hundred Citation Classics in General Surgical Journals. World J Surg. 2002;26:1099-105. 